\title{
Two series of new semisynthetic triterpene derivatives: differences in anti-malarial activity, cytotoxicity and mechanism of action
}

\author{
Gloria NS da Silva ${ }^{1,2}$, Nicole RG Maria', Desirée C Schuck², Laura N Cruz ${ }^{2}$, Miriam S de Moraes², Myna Nakabashi², \\ Cedric Graebin³, Grace Gosmann¹, Célia RS Garcia² and Simone CB Gnoatto ${ }^{1 *}$
}

\begin{abstract}
Background: The discovery and development of anti-malarial compounds of plant origin and semisynthetic derivatives thereof, such as quinine (QN) and chloroquine (CQ), has highlighted the importance of these compounds in the treatment of malaria. Ursolic acid analogues bearing an acetyl group at C-3 have demonstrated significant anti-malarial activity. With this in mind, two new series of betulinic acid (BA) and ursolic acid (UA) derivatives with ester groups at C-3 were synthesized in an attempt to improve anti-malarial activity, reduce cytotoxicity, and search for new targets. In vitro activity against CQ-sensitive Plasmodium falciparum 3D7 and an evaluation of cytotoxicity in a mammalian cell line (HEK293T) are reported. Furthermore, two possible mechanisms of action of anti-malarial compounds have been evaluated: effects on mitochondrial membrane potential $\left(\Delta \psi_{m}\right)$ and inhibition of $\beta$-haematin formation.
\end{abstract}

Results: Among the 18 derivatives synthesized, those having shorter side chains were most effective against CQsensitive $P$. falciparum 3D7, and were non-cytotoxic. These derivatives were three to five times more active than BA and $\cup A$. $A D i_{6}(3) \Delta \psi m$ assay showed that mitochondria are not involved in their mechanism of action. Inhibition of $\beta$-haematin formation by the active derivatives was weaker than with CQ. Compounds of the BA series were generally more active against $P$. falciparum 3D7 than those of the UA series.

Conclusions: Three new anti-malarial prototypes were obtained from natural sources through an easy and relatively inexpensive synthesis. They represent an alternative for new lead compounds for anti-malarial chemotherapy.

Keywords: Plasmodium falciparum, Anti-malarial, Mitochondrial membrane potential, $\beta$-haematin, Betulinic acid, Ursolic acid, Semisynthesis

\section{Background}

Malaria is among the most significant of infectious diseases, influencing the health of humankind to this day [1]. Plasmodium falciparum is a protozoan parasite and the causative agent of the most virulent form of malaria in humans. In endemic areas, malaria accounts for nearly one million deaths, primarily among children under the age of five [2].

\footnotetext{
*Correspondence: simone.gnoatto@ufrgs.br

'Phytochemistry and Organic Synthesis Laboratory, School of Pharmacy, Federal University of Rio Grande do Sul, Porto Alegre 90610-000, Brazil Full list of author information is available at the end of the article
}

Higher plants are commonly used as sources for the discovery of new drug leads. Quinine $(\mathrm{QN})$ and artemisinin are examples of plant-derived products with anti-malarial activity [3]. QN, one of the oldest and most important anti-malarial agents, has long been extracted from the bark of Cinchona (Rubiaceae) species [4]. Many other natural and semisynthetic anti-malarial compounds have since been developed, most of which fall into three classes: the quinolines, the artemisinin-based anti-malarials and the antifolates [5]. Nevertheless, due to the emergence of resistant Plasmodium strains, the therapeutic impact of these compounds has declined, urging development of new and effective anti-malarials [6]. The lack of low-cost

\section{() Biomed Central}


and non-toxic drugs creates a very disturbing scenario for malaria management, and greater efforts must be made toward the discovery of new anti-malarial compounds [7]. The natural compound betulinic acid (3ß-hydroxy-lup-20 (29)-en-28-oic acid, BA) has been shown to exhibit a variety of biological activities, including inhibition of human immunodeficiency virus (HIV), antibacterial, anti-inflammatory, anticancer and anti-helminthic effects $[8,9]$. The in vitro anti-plasmodial activity $\left(\mathrm{IC}_{50}\right)$ of $\mathrm{BA}$ against chloroquine (CQ)-resistant (K1) and CQ-sensitive (T9-96) P. falciparum strains was found to be $19.6 \mu \mathrm{g} / \mathrm{mL}$ and $25.9 \mu \mathrm{g} / \mathrm{mL}$, respectively. In a murine malaria model using Plasmodium berghei NK65 strain, BA exhibited some toxicity when a high dosage was employed ( $250 \mathrm{mg} / \mathrm{kg} /$ day $)$, but no toxicity was observed at a dose of $125 \mathrm{mg} / \mathrm{kg} /$ day [10].

Another triterpene of pharmacological importance is ursolic acid (3ß-hydroxyurs-12-en-28-oic acid, UA), which is known to have anti-inflammatory and antihyperlipidemic properties as well as antitumour-promoting effects [11]. Moreover, UA has been shown to suppress parasitaemia (96.9\% at $60 \mathrm{mg} / \mathrm{kg} /$ day) in $P$. berghei-infected mice and reduce in vitro proliferation of $P$. falciparum strains 3D7, W2, and K1 [10,12]. The mechanism of action of triterpenes as anti-malarials is not fully understood. Gnoatto et al. [13] described seven new ursolic acid analogues bearing an acetyl group at C-3 and a piperazine moiety at $\mathrm{C}-28$ with significant antimalarial activity in the nanomolar range. The hydrophobic interactions of the UA skeleton were also characterized through molecular dynamic simulations, supporting the binding of triterpene to haem as a mechanism of action for these new UA derivatives. Therefore, the search for new triterpene derivatives is a promising approach for development of drugs with potential anti-malarial activity (Figure 1).

In this context, the present study report the synthesis and anti-malarial activity (against CQ-sensitive P. falciparum 3D7) of two new series of BA and UA analogues possessing various ester substituents at $\mathrm{C}-3$, as well as the in vitro cytotoxicity of the most active compounds in
HEK293T mammalian cells and their effect on mitochondrial membrane potential $(\Delta \Psi m)$ and inhibition of $\beta$-haematin formation. These mechanisms of action are likely involved in the anti-malarial activity of atovaquone and CQ respectively $[14,15]$.

\section{Methods}

Collection of plant materials and isolation of BA and UA

Platanus acerifolia bark was collected in Porto Alegre, RS, Brazil. After botanical identification, a voucher specimen, ICN 171329, was deposited in the Herbarium of the Federal University of Rio Grande do Sul Department of Botany. BA was isolated as described in the additional material (Additional file 1) and its structure was confirmed using spectroscopic data and comparison with the existing literature [16]. UA was isolated from waste apple (Malus domestica) peels obtained from a local juice factory. This compound was identified using full spectroscopy data, which consistent with those previously described [13].

\section{Semisynthesis of the BA and UA series}

All commercially available reagents were used without further purification unless otherwise stated. Solvents were distilled under a positive-pressure dry nitrogen atmosphere when necessary. Reactions requiring anhydrous conditions were performed under a nitrogen atmosphere. $\mathrm{BA}$ and UA derivatives were synthesized according to the general procedure described in the literature [17]. Briefly, the appropriate anhydride (1.1 mmol, $5 \mathrm{Eq})$ and DMAP (0.22 mmol, $1 \mathrm{Eq})$ were added to BA or UA $(0.22 \mathrm{mmol})$ in pyridine or $\mathrm{CH}_{2} \mathrm{Cl}_{2}(2 \mathrm{~mL})$ and refluxed for 24 hours (for cyclic anhydrides) or processed without refluxing for one hour (for acyclic anhydrides). Column chromatography of the crude residue was performed to give the expected pure compounds. Series BA and UA are illustrated in Figure 2.

Column chromatography was carried out using silica gel 60 (Merck). Analytical thin layer chromatography was performed on silica gel $60 \mathrm{~F}_{254}$ plates (Merck) and spots visualized by spraying with anisaldehyde/sulphuric

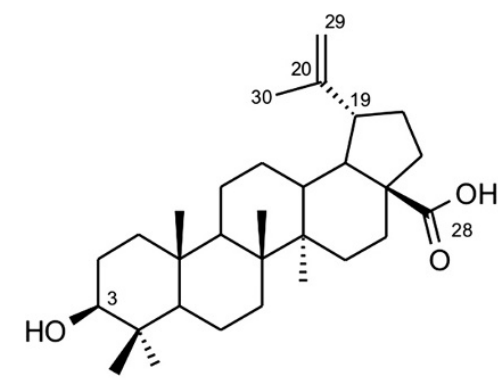

BA

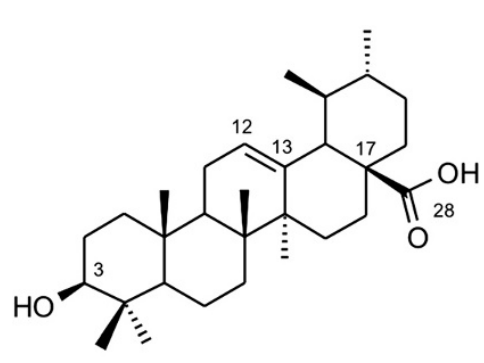

UA

Figure 1 Betulinic (BA) and ursolic (UA) acids. 


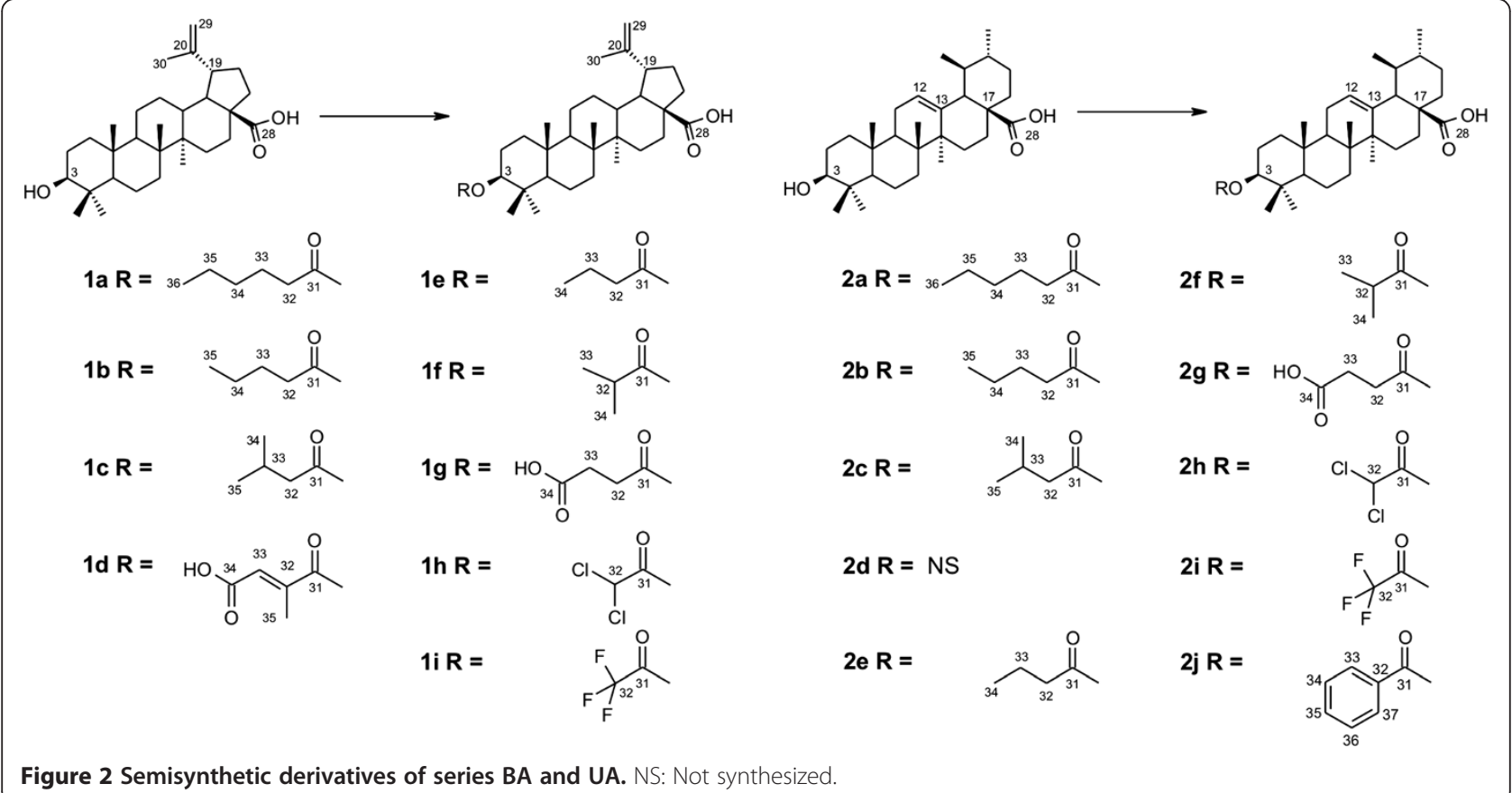

acid solution followed by heating at $100^{\circ} \mathrm{C}$. Melting points were determined using a Kofler bench. Highresolution mass spectra (HR-EI-MS) were obtained on a Micromass-Waters Q-TOF Ultima spectrometer. Infrared spectra were recorded on a PerkinElmer FTIR BX spectrometer. ${ }^{1} \mathrm{H}$ and ${ }^{13} \mathrm{C}$ Nuclear Magnetic Resonance (NMR) spectra were recorded on a Bruker AC 400 spectrometer operating at 300 and $75 \mathrm{MHz}$, respectively, using tetramethylsilane as the internal standard and chloroform-d. The chemical shifts $(\delta)$ were expressed in parts per million (ppm).

\section{Determination of in vitro anti-malarial activity}

Plasmodium falciparum (3D7 strain) was cultured in RPMI 1640 supplemented with 37.5 mM HEPES, $7 \mathrm{mM}$ D-glucose, $6 \mathrm{mM} \mathrm{NaOH}, 25 \mu \mathrm{g} / \mathrm{mL}$ gentamicin sulfate, $2 \mathrm{mM} \mathrm{L}$-glutamine and 10\% human serum and maintained in human erythrocytes under a gas mixture of $5 \% \mathrm{O}_{2}, 5 \%$ $\mathrm{CO}_{2}$, and $90 \% \mathrm{~N}_{2}$ [18]. Culture was synchronized by using $10 \%$ sorbitol [19]. Infected red blood cells (iRBC) with a parasitaemia of $1-2 \%$ and a haematocrit of $2 \%$ were incubated with compounds over a concentration range of 0.001 to $100 \mu \mathrm{M}$ for 48 hours. CQ was included as positive control and the negative control was the solvent. Stock solutions of CQ and test compounds, prepared in water and dimethylsulfoxide (DMSO) respectively, were serially diluted with culture medium and added to synchronous parasite cultures in 96-well plates, following the addition of $200 \mu \mathrm{L}$ formaldehyde solution $2 \%$ per well, overnight. Parasitaemia was evaluated by Giemsa-stained smears and flow cytometry. iRBCs were stained with $\mathrm{YOYO}^{\circledR}-1$. The concentration required to inhibit parasite growth was determined by comparing the fluorescence of the treated and untreated (control) cultures. The concentration causing $50 \%$ inhibition $\left(\mathrm{IC}_{50}\right)$ was obtained from the drug concentration response curve. The DMSO concentration never exceeded $0.1 \%$ and did not inhibit parasite growth. The definition of anti-malarial activity used herein was: $\mathrm{IC}_{50} \leq 1$ $\mu \mathrm{M}$, potent activity; $\mathrm{IC}_{50} 1-10 \mu \mathrm{M}$, good activity; $\mathrm{IC}_{50} 10$ $30 \mu \mathrm{M}$, moderate activity; $\mathrm{IC}_{50} \geq 30 \mu \mathrm{M}$, inactive [20]. Reagents were purchased from Merck and Sigma-Aldrich.

\section{In vitro cytotoxicity}

Cytotoxicity was estimated using HEK293T (human embryonic kidney) cells. This cell line was cultured in $75 \mathrm{sq}$ $\mathrm{cm}$ vented tissue culture flasks at $37^{\circ} \mathrm{C}$ in a humidified atmosphere containing $5 \% \mathrm{CO}_{2}$, in Dulbecco's modified essential medium (Gibco BRL) supplemented with 10\% (v/v) foetal bovine serum, $100 \mathrm{U} / \mathrm{mL}$ penicillin, and $100 \mu \mathrm{g} / \mathrm{mL}$ streptomycin. Cells $\left(5.0 \times 10^{4} /\right.$ well $)$ were seeded into 48-well plates. Twenty-four hours after plating, cells were treated with decreasing concentrations (100; $10 ; 1 ; 0.1 ; 0.01$ and $0.001 \mu \mathrm{M}$ ) of compounds $1 \mathbf{1 e}, \mathbf{1 f}$ and $2 \mathbf{e}$ and evaluated after 24 and 48 hours. After incubation, cells were harvested with trypsin, gently centrifuged and resuspended in phosphate buffer saline. The cells were stained with dihydroethidium solution $(10 \mathrm{mg} / \mathrm{mL}$ in phosphate buffered saline), gently vortexed, and incubated for $40 \mathrm{~min}$ at $37^{\circ} \mathrm{C}$ in the dark. Ten thousand gated events were acquired for each sample (FACSCalibur, Becton \& Dickinson, and FlowJo software). The percentage of cell viability calculated as intrinsic cytotoxicity is represented 
by the concentration leading to $50 \%$ of cell death $\left(\mathrm{IC}_{50}\right)$ on flow cytometry.

\section{Determination of changes in mitochondrial membrane potential $(\boldsymbol{\Delta} \Psi \boldsymbol{m})$}

Changes in mitochondrial membrane potential were assessed as per Srivastava et al. [14]. Accumulation of the lipophilic cationic fluorescent probe 3,3'-dihexyloxacarb ocyanine iodide $\left(\mathrm{DiOC}_{6}(3)\right)$ in mitochondria was assessed in the presence of the active compounds $\mathbf{1 e}, \mathbf{1 f}$ and $\mathbf{2 e}$ and in their absence, in comparison with cells treated with the mitochondrial respiratory chain uncoupler carbonyl cyanide $m$-chlorophenyl hydrazone (CCCP). In this manner, iRBC in RPMI 1640 medium containing 10\% human serum and $10 \%$ parasitaemia were incubated with a $2 \mathrm{nM}$ final concentration of $\mathrm{DiOC}_{6}(3)$ for $30 \mathrm{~min}$ at $37^{\circ} \mathrm{C}$. Various concentrations of the compounds were then added to the culture and incubated for an additional $30 \mathrm{~min}$ at $37^{\circ} \mathrm{C}$. At the end of the incubation period, samples were centrifuged for $5 \mathrm{~min}$ at 3,700 rpm and the supernatant discarded. Samples were then resuspended in phosphate buffer saline and $10^{5}$ events analyzed by flow cytometry.

\section{Inhibition of $\beta$-haematin formation}

Inhibition of $\beta$-haematin formation was determined as described by Baelmens et al. [21]. CQ was used as the positive control. Briefly, $100 \mu \mathrm{L}$ of a fresh $6.5 \mathrm{mM}$ solution of haemin in $0.2 \mathrm{M} \mathrm{NaOH}$ was mixed with $200 \mu \mathrm{L}$ of $3 \mathrm{M}$ sodium acetate, $25 \mu \mathrm{L}$ of $17.4 \mathrm{M}$ acetic acid and $25 \mu \mathrm{L}$ of the tested compound or the solvent as negative control (DMSO for $\mathbf{1 e}$, 1 f and $\mathbf{2 e}$ or water for CQ). The final concentration of compounds in wells ranged from 0.5 to 20 $\mathrm{mM}$. After 24 hours of incubation while gently shaking at $37^{\circ} \mathrm{C}$, samples were centrifuged for $15 \mathrm{~min}$ at 3,300 $\mathrm{g}$, the supernatant discarded and the pellet washed with $200 \mu \mathrm{L}$ DMSO. This latter step was repeated once more and the pellet was finally washed with water. The pellet was then dissolved in $200 \mu \mathrm{L}$ of $0.1 \mathrm{M} \mathrm{NaOH}$. After a further 1:8 dilution, absorption at $405 \mathrm{~nm}$ was measured using a FlexStation plate reader. Experiments were carried out at least in triplicate. Results are expressed as percentage of inhibition of $\beta$-haematin formation in comparison to the negative control result, as calculated with the equation

$\%$ Inhibition $=100 *(1-($ OD drug $) /($ OD solvent $))$

\section{Statistical analysis}

All results are expressed as mean \pm standard error or mean \pm standard deviation of at least three individual experiments. Student's $t$ test was chosen for betweengroup comparisons, whereas repeated measures ANOVA was used for comparisons amongst more than two groups. Statistical significance was defined as $p<0.05$. Analyses were carried out in GraphPad Prism version 4.00 for Windows (GraphPad Software, San Diego, CA USA).

\section{Results}

\section{Description of natural triterpenes and their synthetic} derivatives

The structures of the isolated compounds are shown in Figure 1, and their synthetic derivatives are shown in Figure 2.

The triterpene BA was isolated from $P$. acerifolia bark, and UA from $M$. domestica peel; both sources were highly advantageous due to their low cost and good yields ( $2 \%$ for BA and $2.8 \%$ for UA; expressed in \% weight/weight of dried bark or raw material). After semisynthesis of the eighteen compounds in series BA and UA, their structural elucidation could be deduced from IR, ${ }^{1} \mathrm{H},{ }^{13} \mathrm{C}$ NMR, HR-EI-MS and elemental analysis data (Additional file 2).

\section{Plasmodium falciparum susceptibility}

Table 1 presents the $\mathrm{IC}_{50}$ for the isolated and synthesized compounds against CQ-sensitive $P$. falciparum $3 \mathrm{D} 7\left(\mathrm{IC}_{50} \mathrm{CQ}=29 \mathrm{nM}\right)$. The $\mathrm{IC}_{50}$ for $\mathrm{BA}$ and UA were 18 and $36 \mu \mathrm{M}$ respectively, which is consistent with previously published data [12,22]. Compounds 1e, 1f, and 2e showed good activity and BA, $\mathbf{1 b}$, and $\mathbf{1 h}$ showed moderate activity, whereas the other compounds, including UA, were considered inactive.

\section{Cytotoxicity of active compounds on HEK293T mammalian cells}

The $\mathrm{IC}_{50}$ of compounds $\mathbf{1 e}$, 1 and $2 \mathbf{e}$ for HEK293T was $>100 \mu \mathrm{M}$; therefore, none was cytotoxic in the tested conditions.

\section{Determination of changes in mitochondrial membrane potential $(\boldsymbol{\Delta \Psi \boldsymbol { m } )}$}

Figure 3A presents the representative fluorescence histogram for iRBC displacement in incubation with CCCP at a concentration of $5 \mu \mathrm{M}$, indicating a differential probe location and ideal experimental conditions. Histograms obtained in control conditions (Figure 3B) and in the presence of the active compounds 1e, 1f and $\mathbf{2 e}$ (Figure 3C, 3D and 3E, respectively) showed a normal mitochondrial membrane potential for the concentrations tested, in the $0.25 \mathrm{nM}-25 \mu \mathrm{M}$ range. These results suggest that the mechanism of anti-malarial action of these compounds in $P$. falciparum iRBC is distinct from that of atovaquone. Results were calculated from fluorescence intensity by flow cytometer setting.

\section{Inhibition of $\beta$-haematin formation}

Inhibition percentages for the most active derivatives are reported in Table 2. CQ was significantly more efficient at inhibiting $\beta$-haematin formation than all tested compounds. 
Table 1 Anti-malarial activity of isolated and synthesized compounds against CQ-sensitive Plasmodium falciparum 3D7

\begin{tabular}{llll}
\hline Series BA & \multicolumn{3}{c}{ Series UA } \\
\hline Compound & $\mathbf{I C}_{\mathbf{5 0}}{ }^{\mathbf{a}}(\boldsymbol{\mu M})$ & Compound & $\mathbf{I C}_{\mathbf{5 0}}(\boldsymbol{\mu M})$ \\
\hline $\mathrm{BA}$ & $18 \pm 0.17$ & $\mathrm{UA}$ & $36 \pm 0.25$ \\
$\mathbf{1 a}$ & $>100$ & $\mathbf{2 a}$ & $>100$ \\
$\mathbf{1 b}$ & $29 \pm 0.26$ & $\mathbf{2 b}$ & $71 \pm 0.30$ \\
$\mathbf{1 c}$ & $44 \pm 0.14$ & $\mathbf{2 c}$ & $72 \pm 0.16$ \\
$\mathbf{1 d}$ & $70 \pm 0.19$ & $\mathbf{2 d}$ & $\mathrm{ND}$ \\
$\mathbf{1 e}$ & $5 \pm 0.14$ & $\mathbf{2 e}$ & $7 \pm 0.15$ \\
$\mathbf{1 f}$ & $8 \pm 0.16$ & $\mathbf{2 f}$ & $>100$ \\
$\mathbf{1 g}$ & $66 \pm 0.25$ & $\mathbf{2 g}$ & $58 \pm 0.13$ \\
$\mathbf{1 h}$ & $22 \pm 0.17$ & $\mathbf{2 h}$ & $58 \pm 0.32$ \\
$\mathbf{1 i}$ & $>100$ & $\mathbf{2 i}$ & $>100$ \\
$\mathbf{1 j}$ & $\mathrm{ND}$ & $\mathbf{2 j}$ & $>100$ \\
\hline
\end{tabular}

$\mathrm{a}_{\text {: }}$ results expressed as mean \pm standard error.

ND Not Determined;

$B A$ Betulinic Acid.

UA Ursolic Acid.
No statistically significant differences were found between 1e and 2e (derived from BA and UA respectively). This suggests that the tested derivatives exert their anti-malarial effects via a mechanism of action distinct from that of $C Q$ in the haemin detoxification pathway.

\section{Discussion}

In this study, semisynthetic compounds were obtained, derived from natural sources, with potential utility as antimalarial agents, using a simple and inexpensive strategy.

The triterpenes BA and UA exhibited moderate antimalarial activity in vitro $[12,22]$. Both compounds were obtained from natural sources with good yields. This work reported for the first time the semisynthesis of 18 BA and UA derivatives with ester substituents at C-3, designed to improve anti-malarial activity, reduce cytotoxicity and search for new targets. These derivatives were obtained using a single-step, inexpensive synthesis adequate for industrial-scale processing. The anti-malarial activities of the synthesized compounds were in the range of 5 and $>100 \mu \mathrm{M}$ against CQ-sensitive P. falciparum

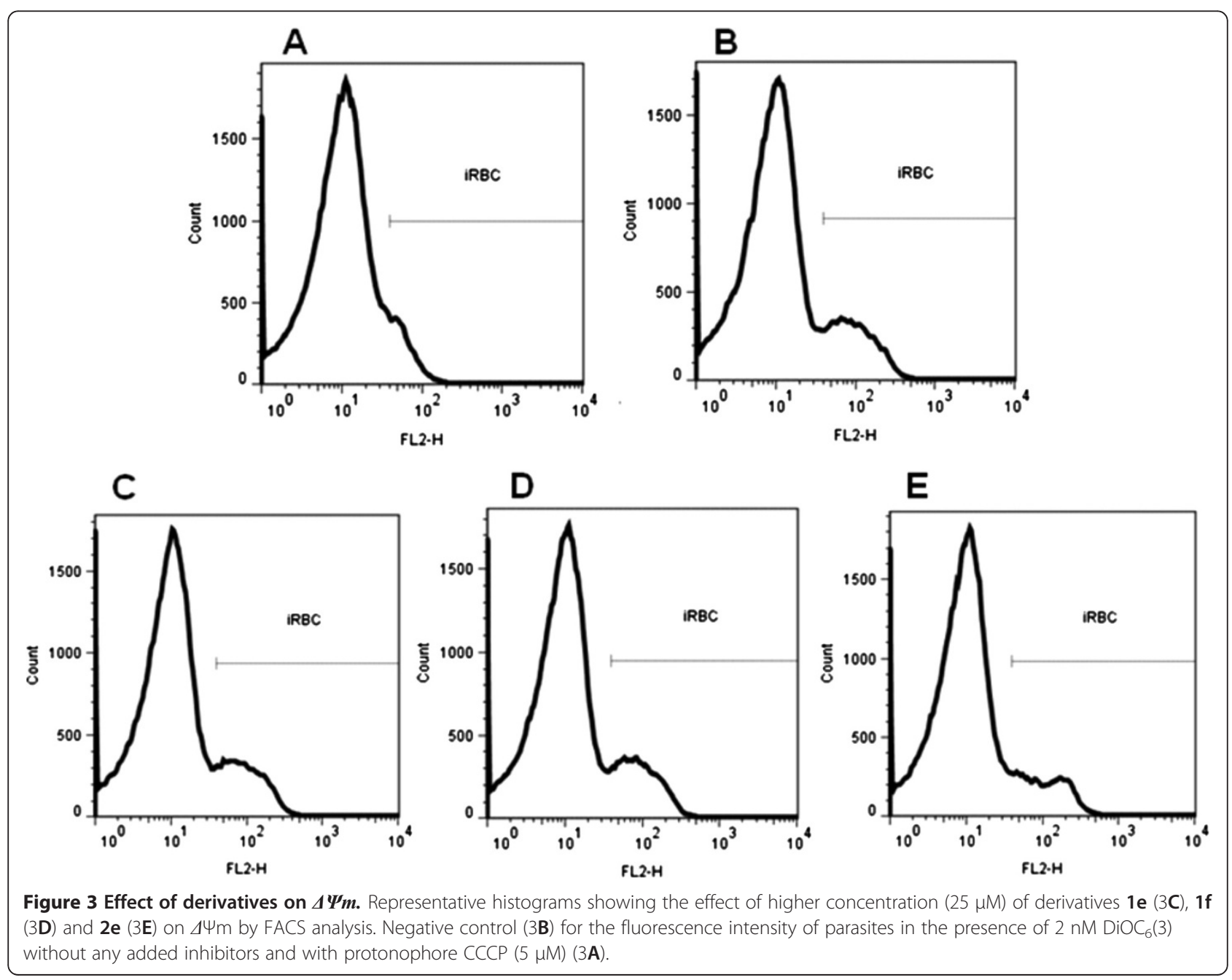


Table 2 Inhibition of $\boldsymbol{\beta}$-haematin formation

\begin{tabular}{lllll}
\hline Compound & $\begin{array}{l}\text { Concentration } \\
(\mathbf{m M})\end{array}$ & $\begin{array}{l}\text { Inhibition }^{\mathrm{b}} \\
\mathbf{( \% )}\end{array}$ & $\begin{array}{l}\mathrm{IC}_{\mathbf{5 0}}{ }^{\mathrm{C}} \\
(\mathbf{m M})\end{array}$ & $\begin{array}{l}\mathrm{CQ} \\
\text { index }^{\mathrm{a}}\end{array}$ \\
\hline Chloroquine & $0.5-20$ & $83 \pm 1.07$ & $3 \pm 0.16$ & - \\
1e & $0.5-19$ & $25 \pm 2.62$ & $>19$ & $>6.33$ \\
1f & $0.5-20$ & $14 \pm 0.43$ & $>20$ & $>6.66$ \\
2e & $0.5-19$ & $26 \pm 3.57$ & $>19$ & $>6.33$
\end{tabular}

${ }^{a}$ calculated as compound $\mathrm{IC}_{50} / \mathrm{CQ} \mathrm{IC}_{50}$.

${ }^{b}$ results are expressed as mean \pm standard deviation at the highest tested concentration.

c results are expressed as mean \pm standard error.

3D7, with three compounds exhibiting good activity (1e, 1f and 2e). These three derivatives possessed a four-carbon side-chain and had anti-malarial activity three to five times greater than that of BA and UA. Some reports have reported a change in activity after modification on the triterpene carbon 3. The 3-O-acetyl ester of ursolic acid exhibited enhanced activity against CQ-resistant $P$. falciparum $\mathrm{FcB} 1\left(\mathrm{IC}_{50}=\right.$ $24.93 \mu \mathrm{M})$ as compared with UA $\left(\mathrm{IC}_{50}=52.93 \mu \mathrm{M}\right)$ [13]. Interestingly, the 3-O-acetyl ester of oleanolic acid, an isomer of UA, was also active against a multidrug-resistant P. falciparum K1 strain [23]. In another study, the 3-O -acetyl ester of lupeol containing a functional ester group at C-3 was more active than its precursor against CQresistant $P$. falciparum FCR-3 [24]. In view of these findings, modifications at the $\mathrm{C}-3$ position of $\mathrm{BA}$ and UA were designed to evaluate the influence of side-chain length and presence of polar or lipophilic groups (such as carboxylic acids, aromatic rings or halogens) on their ability to impair parasite growth. In these series, acylated derivatives with shorter side-chains (1e, 1f and 2e) had improved antimalarial activity $\left(\mathrm{IC}_{50}=5,8\right.$ and $7 \mu \mathrm{M}$, respectively). It was also obtained information on the presence of a second carboxylic acid group, as in derivatives $\mathbf{1 d}\left(\mathrm{IC}_{50}=70 \mu \mathrm{M}\right), \mathbf{1 g}$ $\left(\mathrm{IC}_{50}=66 \mu \mathrm{M}\right)$ and $2 \mathrm{~g}\left(\mathrm{IC}_{50}=58 \mu \mathrm{M}\right)$; and the presence of a halogen, such as chlorine and fluorine, as in derivatives 1h $\left(\mathrm{IC}_{50}=22 \mu \mathrm{M}\right), \mathbf{1 i}\left(\mathrm{IC}_{50}>100 \mu \mathrm{M}\right), \mathbf{2 h}\left(\mathrm{IC}_{50}=58 \mu \mathrm{M}\right)$ and $2 \mathbf{i}\left(\mathrm{IC}_{50}>100 \mu \mathrm{M}\right)$. These functional groups did not potentiate anti-malarial action.

Comparison of derivatives with the same substituents derived from series BA and series UA demonstrated that BA derivatives are generally more active against $P$. falciparum 3D7 strain than UA derivatives. The most active derivatives synthesized, $\mathbf{1 e}, \mathbf{1 f}$ and $\mathbf{2 e}$, did not show any cytotoxicity against HEK293T cells at the tested concentration of $100 \mu \mathrm{M}$. This excellent result justifies potential in vivo experiments with these compounds in future. Follow-up studies with non-cytotoxic compounds are important, as adverse effects such as hypoglycaemia, cardiotoxicity, and gastrointestinal discomfort have been described with the anti-malarials: QN, halofantrine, and $\mathrm{CQ} /$ proguanil [22].
The molecular mechanism of action of triterpenes such as BA and UA is still poorly understood. Previous research has suggested that the mechanism of action of $\mathrm{UA}$ and its derivatives could be similar to that of $\mathrm{CQ}$, i.e. inhibition of $\beta$-haematin formation [13,25]. It was chose to assess this mechanism of action and another mechanism that has been suggested for an important antimalarial agent, atovaquone: an inhibitory effect on $\Delta \Psi m$. The $\Delta \Psi m$ consists of chemical and electrical components generated by electron transport chain enzymes, and its determination is widely used to characterize cellular metabolism, viability and apoptosis [26]. The $\Delta \Psi m$ assay was performed for the more active derivatives, $\mathbf{1 e}$, 1f and 2e, by accumulation of the lipophilic cationic fluorescent probe $\operatorname{DiOC}_{6}(3)$. When $\mathrm{DiOC}_{6}(3)$ is incubated with iRBC, it diffuses into cells and concentrates several orders of magnitude into negative-inside mitochondria. Probe accumulation into parasite mitochondria is dependent on the presence of a membrane potential, collapse of which will result in diffusion of the probe out of the mitochondria, resulting in signal dissipation. The tested derivatives did not exhibit histogram displacement, as observed after incubation with CCCP, indicating dissipation of the membrane potential and abolition of probe accumulation. This experiment showed that, unlike CCCP, the tested compounds were not able to collapse $\Delta \Psi m$ (Figure 3).

Derivatives 1e, 1 f and 2e were also evaluated for inhibition of $\beta$-haematin formation, and displayed less inhibitory activity than that of CQ. This assay is based on the ability of Plasmodium to use haemoglobin as a source of amino acids, resulting in the formation of potentially toxic ferriprotoporphyrin IX. CQ and other anti-malarial drugs act by inhibiting ferriprotoporphyrin IX detoxification through haem polymerization. The action of these antimalarial drugs on $\beta$-haematin formation takes place during the intra-erythrocytic phase of the parasite, within the food vacuole. The parasite converts haem into the malarial pigment haemozoin [15]. Derivatives $1 \mathbf{e}$ and 2e derivatives exhibited similar inhibition percentages (25\% and $26 \%$ respectively), whereas 1f displayed less inhibitory activity (14\%) than the other tested derivatives. Thus, it was observed that modification of carbon 3 plays an important role in the inhibition of $\beta$-haematin formation.

\section{Conclusion}

In this study, 18 new derivatives of betulinic acid (series BA) and ursolic acid (series UA) were synthesized with the purpose of investigating the importance of substituents at carbon 3 for anti-malarial activity against $P$. falciparum 3D7. Derivatives 1e, $\mathbf{1 f}$ and $\mathbf{2 e}$ exhibited good anti-malarial activity in the micromolar range. Derivatives of series BA were generally more active against $P$. falciparum $3 \mathrm{D} 7$ than compounds derived from series UA, with a three- to five- 
fold difference in activity in relation to their respective aglycones. Furthermore, 1e, 1f and 2e did not exhibit any cytotoxicity against a HEK293T cell line at $100 \mu \mathrm{M}$. Regarding mechanism of action, these triterpene derivatives was weaker inhibit $\beta$-haematin formation than CQ and did not act on $\Delta \Psi m$. Further assays are required to elucidate the pathways whereby these anti-malarial compounds exert their effects. This study can contribute to the design and development of potent anti-malarial compounds derived from natural sources.

\section{Additional files}

Additional file 1: Extraction of BA and UA. Description: The data provided represent the extraction of betulinic and ursolic acids.

Additional file 2: Identification of synthesized derivatives.

Description: The data provided represent the identification of the synthesized derivatives

\section{Abbreviations}

BA: Betulinic acid; CQ: Chloroquine; OD: Optical density; UA: Ursolic acid.

\section{Competing interests}

The authors declare that they have no competing interests.

\section{Authors' contributions}

SCBG, GG, CRSG and GNSS designed and coordinated the research. GNSS and NRGM obtained the betulinic and ursolic acids, and corresponding derivatives. GNSS and MN performed the experiments for the anti-malaria activity. GNSS and MSM performed the cytotoxicity assays. GNSS, DCS and LNC were responsible for the assay on $\Delta \psi_{m}$ and inhibition of $\beta$-haematin formation. GNSS and LNC drafted the manuscript. SCBG, GG and CRSG corrected the manuscript. All authors approved the final manuscript.

\section{Acknowledgements}

This work was supported by grants and financial support from Brazilian Agencies CAPES, CNPq, INCT-IF, Malaria-PRONEX, INCT-INBEQMeDI, FAPESP and the Graduate Programme in Pharmaceutical Sciences (PPGCF/UFRGS). The authors are also grateful to the apple samples from Tecnovin do Brasil LTDA, RS, Brazil. We thank Prof Dr N P Lopes (USP-RP) for HRMS analysis. LNC and MSM received FAPESP Fellowships.

\section{Author details}

${ }^{1}$ Phytochemistry and Organic Synthesis Laboratory, School of Pharmacy, Federal University of Rio Grande do Sul, Porto Alegre 90610-000, Brazil. 2Plasmodium Molecular and Cellular Biology Laboratory, Department of Physiology, São Paulo University, São Paulo 05508-900, Brazil. ${ }^{3}$ Department of Chemistry, Federal Rural University of Rio de Janeiro, Seropedica 23897/000, Brazil.

Received: 9 October 2012 Accepted: 2 March 2013

Published: 9 March 2013

\section{References}

1. Pasternak ND, Dzikowski R: PfEMP1: an antigen that plays a key role in the pathogenicity and immune evasion of the malaria parasite Plasmodium falciparum. Int J Biochem Cell Biol 2009, 41:1463-1466.

2. Baumeister $S$, Winterberg M, Przyborski JM, Lingelbach K: The malaria parasite Plasmodium falciparum: cell biological peculiarities and nutritional consequences. Protoplasma 2010, 240:3-12

3. Kayser $\mathrm{O}$, Kiderlen AF, Croft SL: Natural products as antiparasitic drugs. Parasitol Res 2003, 90(Suppl 2):S55-S62.

4. Baird JK, Caneta-Miguel E, Masbar S, Bustos DG, Abrenica JA, Layawen AV, Calulut JM, Leksana B, Wignall FS: Survey of resistance to chloroquine of falciparum and vivax malaria in Palawan, The Philippines. Trans $R$ Soc Trop Med Hyg 1996, 90:413-414.
5. Carmargo LM, De Oliveira S, Basano S, Garcia CR: Antimalarials and the fight against malaria in Brazil. Ther Clin Risk Manag 2009, 5:311-317.

6. Biot C, Chibale K: Novel approaches to antimalarial drug discovery. Infect Disord Drug Targets 2006, 6:173-204

7. Zofou D, Kowa TK, Wabo HK, Ngemenya MN, Tane P, Titanji VP: Hypericum lanceolatum (Hypericaceae) as a potential source of new anti-malarial agents: a bioassay-guided fractionation of the stem bark. Malar J 2011, 10:167.

8. Yogeeswari P, Sriram D: Betulinic acid and its derivatives: a review on their biological properties. Curr Med Chem 2005, 12:657-666.

9. Qian K, Kim SY, Hung HY, Huang L, Chen CH, Lee KH: New betulinic acid derivatives as potent proteasome inhibitors. Bioorg Med Chem Lett 2011 21:5944-5947

10. Steele JC, Warhurst DC, Kirby GC, Simmonds MS: In vitro and in vivo evaluation of betulinic acid as an antimalarial. Phytother Res 1999, 13:115-119.

11. Liu J: Pharmacology of oleanolic acid and ursolic acid. J Ethnopharmacol 1995, 49:57-68.

12. Traore-Keita F, Gasquet M, Di Giorgio C, Ollivier E, Delmas F, Keita A, Doumbo O, Balansard G, Timon-David P: Antimalarial activity of four plants used in traditional medicine in Mali. Phytother Res 2000, 14:45-47.

13. Gnoatto SC, Susplugas S, Dalla Vechia L, Ferreira TB, Dassonville-Klimpt A, Zimmer KR, Demailly C, Da Nascimento S, Guillon J, Grellier P, Verli H, Gosmann G, Sonnet P: Pharmacomodulation on the 3-acetylursolic acid skeleton: Design, synthesis, and biological evaluation of novel $\mathrm{N}$-\{3-[4-(3-aminopropyl)piperazinyl]propyl\}-3-O-acetylursolamide derivatives as antimalarial agents. Bioorg Med Chem 2008, 16:771-782.

14. Srivastava IK, Rottenberg H, Vaidya AB: Atovaquone, a broad spectrum antiparasitic drug, collapses mitochondrial membrane potential in a malarial parasite. J Biol Chem 1997, 272:3961-3966.

15. Hanscheid T, Egan TJ, Grobusch MP: Haemozoin: from melatonin pigment to drug target, diagnostic tool, and immune modulator. Lancet Infect Dis 2007, 7:675-685

16. Cichewicz RH, Kouzi SA: Chemistry, biological activity, and chemotherapeutic potential of betulinic acid for the prevention and treatment of cancer and HIV infection. Med Res Rev 2004, 24:90-114.

17. Yu D, Sakurai $Y$, Chen $\mathrm{CH}$, Chang FR, Huang L, Kashiwada Y, Lee KH: Anti-AIDS agents 69. Moronic acid and other triterpene derivatives as novel potent anti-HIV agents. J Med Chem 2006, 49:5462-5469.

18. Trager W, Jensen JB: Human malaria parasites in continuous culture. Science 1976, 193:673-675.

19. Lambros C, Vanderberg JP: Synchronization of Plasmodium falciparum erythrocytic stages in culture. J Parasitol 1979, 65:418-420.

20. Ramalhete C, Da Cruz FP, Lopes D, Mulhovo S, Rosario VE, Prudencio M, Ferreira MJ: Triterpenoids as inhibitors of erythrocytic and liver stages of Plasmodium infections. Bioorg Med Chem 2011, 19:7474-7481.

21. Baelmans R, Deharo E, Munoz V, Sauvain M, Ginsburg H: Experimental conditions for testing the inhibitory activity of chloroquine on the formation of beta-hematin. Exp Parasitol 2000, 96:243-248.

22. Duker-Eshun G, Jaroszewski JW, Asomaning WA, Oppong-Boachie F, Brogger Christensen S: Antiplasmodial constituents of Cajanus cajan. Phytother Res 2004, 18:128-130.

23. Kanokmedhakul K, Kanokmedhakul S, Phatchana R: Biological activity of Anthraquinones and Triterpenoids from Prismatomeris fragrans. J Ethnopharmacol 2005, 100:284-288.

24. Fotie J, Bohle DS, Leimanis ML, Georges E, Rukunga G, Nkengfack AE: Lupeol long-chain fatty acid esters with antimalarial activity from Holarrhena floribunda. J Nat Prod 2006, 69:62-67.

25. Mullie C, Jonet A, Dassonville-Klimpt A, Gosmann G, Sonnet P: Inhibitory effect of ursolic acid derivatives on hydrogen peroxide- and glutathionemediated degradation of hemin: a possible additional mechanism of action for antimalarial activity. Exp Parasitol 2010, 125:202-207.

26. Kalbacova M, Vrbacky M, Drahota Z, Melkova Z: Comparison of the effect of mitochondrial inhibitors on mitochondrial membrane potential in two different cell lines using flow cytometry and spectrofluorometry. Cytometry A 2003, 52:110-116.

doi:10.1186/1475-2875-12-89

Cite this article as: Silva et al.: Two series of new semisynthetic triterpene derivatives: differences in anti-malarial activity, cytotoxicity and mechanism of action. Malaria Journal 2013 12:89. 